\title{
Experimental evaluation of electrical properties of fabrics elaborated with conductive yarns
}

\begin{abstract}
In this paper, the electrical characterization of two different fabric structures elaborated with conductive yarns and several pick densities are investigated in order to evaluate their electrical properties as conductive media of digital electrical signals. The real and imaginary impedance and the signal integrity have been measured for each e-textile. The results show that the impedance depends on the fabric structures and pick density. The eye diagram measurements experimentally demonstrate the functionality of these fabrics to transmit digital electric signals to communicate sensors with microcontrollers in wearable applications.
\end{abstract}

Keywords: e-textile, yarn, fabrics, signal integrity, wearable, wireless sensor networks.

\section{Introduction}

In the last years a massive increase of the wireless sensor networks (WSN) has been produced in industrial and domestic applications. The WSN consists of small communication motes. These motes contain a sensing part, a microcontroller, some radiofrequency components, an antenna and power supply (typically a battery) [1]. In this context, many researchers have been paying attention to the integration of these WSN for healthcare applications to monitor physiological (i.e., pulse oximetry, respiration rate, temperature, etc.) and physical behaviour (i.e., limb movements, posture, and muscular activity) [2]. In order to increase the usability and comfort of WSN on wearable healthcare applications, the integration of WSN motes on textile should be addressed.

On the one hand, the integration of electronics in textile substrates has been named electronic textile (e-textile) or smart fabrics. Several papers have been published addressing the design of a specific sensor [3][4][5] or a specific antenna [6] [7] [8] [9] 
on textile substrate and some publications have been devoted to characterize and model the fabrics [10] and demonstrate the functionally of e-textile as an electromagnetic shielding [11]. Most of these research works overlay these electronic devices on the fabrics by means of embroidery textile techniques [12][13] or printed techniques [14]. However, in most cases, these techniques are neither comfortable nor user friendly. In this sense, the integration of electronic devices on the own fabrics would be a more comfortable solution for wearable applications. Therefore, the evaluation of the electrical properties of these electronic devices should be analyzed.

On the other hand, in healthcare applications, the distance between the sensor and the microcontroller can be longer than tens of centimeters. For instance, in an electrocardiogram application, a cable from the electrode located in the ankle to the microcontroller, typically located in hip, should be used. Therefore, if an e-textile is used as a conductive medium for this purpose, the propagation of the signal should be analysed taking into account its temporal behaviour. Moreover, nowadays the communication between sensors and microcontrollers is done typically by means of a serial digital bus, such as the inter-integrated circuit bus (I2C). Thus, the propagation of digital signals on smart fabrics should be evaluated. In this paper, the propagation properties of time dependent electrical signals on several fabrics elaborated with conductive yarn are addressed. In order to do that, the impedance and signal integrity in different cases are experimentally characterized.

The remainder of the paper is organized as follows. Section II describes the fabrics under test as well as the measurement setup. In Section III the experimental results are shown and discussed. Finally, section IV presents the main conclusions. 


\section{Materials and Method}

\section{Technical Fabrics under test}

The fabrics under test (FUT) have been woven by means of a Dornier LWV8/J 190 loom. This loom is equipped with a Jacquard Stäubli LX 1600B 6480-thread electronic machine and it is able to operate at 650 picks/min. In addition, it has a $170 \mathrm{~cm}$ wide reed count of 10 dents/cm and it was threaded in a four yarn/dent.

The fabrics have two different parts based on conductive and non-conductive zones. The non-conductive one was formed for the textile ground, the yarns were used in two different cotton numbers 30/2 Ne (warp) and 40/2 Ne (weft). The density was 38,4 yarns/cm and 15 picks/cm, using 2/2 basket twill like a structure.

On the other hand, the conductive zone was produced with weft silver plated polyamide yarns conductors from Shieldex Ref. 44113 TR Z200 14 (resistivity $<100$ $\Omega / \mathrm{cm})$. It was woven in two different structures: a 2x2 Basket and an irregular twill structure. Fig. 1 shows the SEM image of both structures. In both cases, several thickness of the conductive zone have been evaluated from 13picks/cm to 65 picks/cm (Fig. 2). The total length of the manufactured fabrics is equal to $150 \mathrm{~cm}$. The tested fabrics have been cut at one half of this value. Therefore, a $75 \mathrm{~cm}$ distance between the measurement electrodes has been used. Table 1 summarizes the different configurations studied in this work.

\section{Measurement Setup}

The fabrics under test have been subjected to different measurements in order to analyze their electrical behaviour. First of all, a voltage sweep from $-10 \mathrm{~V}$ to $10 \mathrm{~V}$ of each FUT was measured using a Keithley 2636 source measurement unit to evaluate the DC current-voltage (I-V) behaviour. After that, a Fluke PM6306 LCR meter up to $1 \mathrm{MHz}$ 
was used to characterize their electrical impedance. Finally, the digital signal propagation was evaluated by means of the standard time-domain eye-diagram metric (Fig. 3). This metric is a useful tool for understanding the signal integrity in the physical layer of high-speed digital data systems, verifying transmitter output compliance, and revealing the amplitude and time distortion elements that degrade the propagation performance. The tested eye diagram is the sum of samples from superimposing the 1's, 0's, and corresponding transition measurements. In order to do that, a pseudo-random binary sequence (PRBS) at 4Mbits/s was injected at the input port, whereas the output ports were connected to a Keysight MSO7104A mixed signal oscilloscope. Two parameters were assessed as metrics of the eye-pattern quality: Eye width (EW) and Eye height $(\mathrm{EH})$ as a measure of the horizontal and vertical opening of the eye diagram, respectively.

\section{Results and Discussion}

Fig. 4 shows the DC voltage-current behaviour of the samples i13, i39 and i65, where i denotes IT (Irregular Twill structure) or B (Basket structure). The I-V linearity dependence from $-10 \mathrm{~V}$ to $10 \mathrm{~V}$ is observed for all of them. The current flow increases with the thickness of the FUT for both structures from $129 \mathrm{~mA}$ to $731 \mathrm{~mA}$ for IT13 and IT65, respectively, and from $104 \mathrm{~mA}$ to $557 \mathrm{~mA}$ for B13 and B65, respectively. These results denote that these fabrics can be modelled with a single resistance from the direct current point of view (DC). Moreover, the resistance of the $2 \times 2$ Basket structure is higher than the one of the irregular twill structure for all pick densities.

However, in most cases, the signal to be propagated is a time dependent source. Therefore, the evaluation of the resistance (real part of the impedance) is not enough and the imaginary part of the impedance of the FUT should be determined. 
In Fig. 5 the real and imaginary part of the impedance at $1 \mathrm{MHz}$ is shown. The real impedance increases when the thickness is reduced, as expected according to the resistance law of conductors. Specifically, for the irregular twill structure, the range is extended from $13.83 \Omega$ to $77.7 \Omega$, for IT65 and IT13, respectively. For the basket structure case, the range is observed from $17.95 \Omega$ to $96.15 \Omega$ for B65 and B13, respectively. The basket structures resistance is higher than the corresponding to the irregular twill due to the lower effective metal surface of the textile conductor lines. This fact agrees with the previous results shown in Fig. 4. Moreover, in all cases, the imaginary part is positive. Therefore, it denotes the inductance properties of the fabrics and, as a result, the e-textile can be modelled by means of series RL model, equation 1 .

$$
\mathrm{Z}=\mathrm{R}+\mathrm{j} 2 \pi \mathrm{fL} \quad \Omega
$$

In Table 2 the resistance and inductance per unit length for each structure are reported. Note that, for similar thickness, the resistance of the irregular twill is lower than for the basket structure due to the fact that in the irregular twill the electrical contact between adjacent weft yarns conductors is higher. Otherwise, the inductance of the irregular twill is higher than the basket structure. For the same structure, the resistance and inductance are reduced when the picks/cm are increased. Specifically, the resistance per length is reduced $82.69 \%$ and the inductance per length is reduced $9.06 \%$ from IT13 to IT65. Meanwhile, for the basket structure, from B13 to B65, the resistance and inductance per length are reduced $80.16 \%$ and $40.84 \%$, respectively. These results denote that the resistance is much more pick density sensitive than the inductive component. 
Finally, in order to validate experimentally the functionality of the fabrics elaborated with conductive yarns as a conductive media for I2C digital signals, a signal integrity test has been performed. The eye diagram has been measured at $4 \mathrm{Mbits} / \mathrm{s}$. This bit rate guarantees the highest value of $3.4 \mathrm{Mbits} / \mathrm{s}$ required by the I2C standard. Fig. 6 shows the experimental eye diagram in four corner cases: IT13, IT65, B13 and B65. In all cases, the signal is properly propagated and no significant difference is observed on MEO and MEW, due to the lower inductive impedance at this frequency. Table 3 summarizes the eye diagram parameters for the four cases depicted in Fig. 6. Even though the impedance of both fabric structures is different, the I2C digital signal at 3.4Mbits/s can be correctly propagated in all cases. Therefore, these fabrics elaborated with conductive yarns can be used as a conductive media between sensors and microcontroller on a wearable application.

\section{Conclusions}

Two different fabric structures elaborated with conductive yarns and several pick densities have been electrically characterized. The results show that fabrics' electrical impedance can be modelled as a RL serial electrical model. In all cases, the resistance and inductance increase when the pick density is reduced. Moreover, the impedance can be modified depending on the fabrics structure. The eye diagram measurement shows that these fabrics elaborated with conductive yarns can be used as a propagation medium of I2C digital bus to connect sensors to microcontrollers in e-textile applications. This functionality can help to increase the usability and comfort of wearable technology.

\section{Acknowledgements}

This work was supported by the Spanish Government-MINECO under Grant TEC201341996-R; AGAUR under 2014 SGR 375. 


\section{References}

[1] J. Yick, B. Mukherjee, and D. Ghosal, “Wireless sensor network survey,” Comput. Networks, vol. 52, no. 12, pp. 2292-2330, Aug. 2008.

[2] J. Ko, C. Lu, M. B. Srivastava, J. A. Stankovic, A. Terzis, and M. Welsh, "Wireless Sensor Networks for Healthcare,” Proc. IEEE, vol. 98, no. 11, pp. 1947-1960, Nov. 2010.

[3] J.-S. Roh, “Textile touch sensors for wearable and ubiquitous interfaces,” Text. Res. J., vol. 84, no. 7, pp. 739-750, Nov. 2013.

[4] L. M. Castano and A. B. Flatau, "Smart fabric sensors and e-textile technologies: a review," Smart Mater. Struct., vol. 23, no. 5, p. 053001, May 2014.

[5] G. Cho, K. Jeong, M. J. Paik, Y. Kwun, and M. Sung, "Performance Evaluation of Textile-Based Electrodes and Motion Sensors for Smart Clothing,” IEEE Sens. J., vol. 11, no. 12, pp. 31833193, Dec. 2011.

[6] S. Zhu and R. Langley, “Dual-Band Wearable Textile Antenna on an EBG Substrate,” IEEE Trans. Antennas Propag., vol. 57, no. 4, pp. 926-935, Apr. 2009.

[7] S. Choi and S. Lim, "Foldable thin electro-textile antenna array for $4 \times 4$ multiple-input multipleoutput mobile router applications,” J. Electromagn. Waves Appl., vol. 29, no. 3, pp. 375-385, Jan. 2015.

[8] J.-Y. Kim, S.-J. Ha, D. Kim, B. Lee, and C. W. Jung, "Reconfigurable beam steering antenna using U-slot fabric patch for wrist-wearable applications,” J. Electromagn. Waves Appl., vol. 26, no. 11-12, pp. 1545-1553, Aug. 2012.

[9] T. Kellomäki, J. Virkki, S. Merilampi, and L. Ukkonen, “Towards Washable Wearable Antennas: A Comparison of Coating Materials for Screen-Printed Textile-Based UHF RFID Tags,” Int. J. Antennas Propag., vol. 2012, pp. 1-11, Jan. 2012.

[10] J. Lesnikowski and M. Tokarska, "Modeling of selected electric properties of textile signal lines using neural networks,” Text. Res. J., vol. 84, no. 3, pp. 290-302, Jul. 2013.

[11] C.-W. Lou, A.-P. Chen, C.-W. Lin, W.-H. Hsing, and J.-H. Lin, “Evaluation on manufacturing technique and electromagnetic shielding effectiveness of functional complex fabrics," $J$. Electromagn. Waves Appl., vol. 28, no. 9, pp. 1031-1043, Apr. 2014.

[12] Z. Wang, S. Member, L. Zhang, Y. Bayram, S. Member, and J. L. Volakis, "Embroidered Conductive Fibers on Polymer Composite for Conformal Antennas,” IEEE Trans. Antennas Propag., vol. 60, no. 9, pp. 4141-4147, 2012.

[13] Z. Wang, S. Member, L. Z. Lee, D. Psychoudakis, S. Member, and J. L. Volakis, “Embroidered Multiband Body-Worn Antenna for,” IEEE Trans. Antennas Propag., vol. 62, no. 6, pp. 33213329, 2014.

[14] Yongsang Kim, Hyejung Kim, and Hoi-Jun Yoo, "Electrical Characterization of Screen-Printed Circuits on the Fabric,” IEEE Trans. Adv. Packag., vol. 33, no. 1, pp. 196-205, Feb. 2010. 
Fig.1a. SEM image of 2x2 basket structure fabrics under test.

Fig.1b. SEM image of Irregular twill structure fabrics under test.

Fig.2a. Photograph of 2x2 basket structure with 65 picks/cm.

Fig.2a. Photograph of 2x2 basket structure with 13 picks/cm.

Fig.3. Photograph of experimental setup to evaluate the eye diagram.

Fig.4. DC Voltage current characteristic of FUT.

Fig.5. Real and imaginary impedance of FUT at $1 \mathrm{MHz}$.

Fig. 6a. Eye diagram of FUT IT13.

Fig. 6b. Eye diagram of FUT B13.

Fig. 6c. Eye diagram of FUT IT65.

Fig. 6d. Eye diagram of FUT B65.

Table 1. Fabrics under test.

Table 2. FUT resistance and inductance per unit length.

Table 3. Eye diagram parameter at $4 \mathrm{MHz}$. 


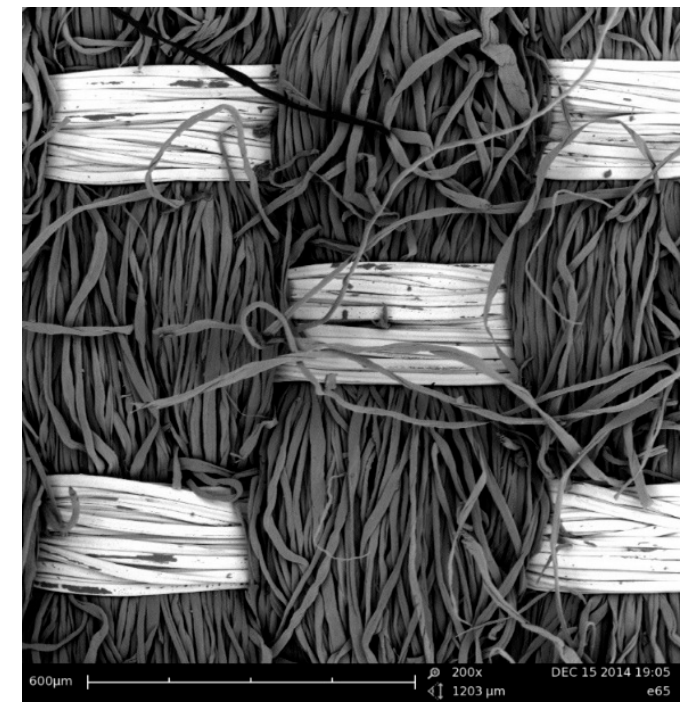

a) $2 \times 2$ basket structure

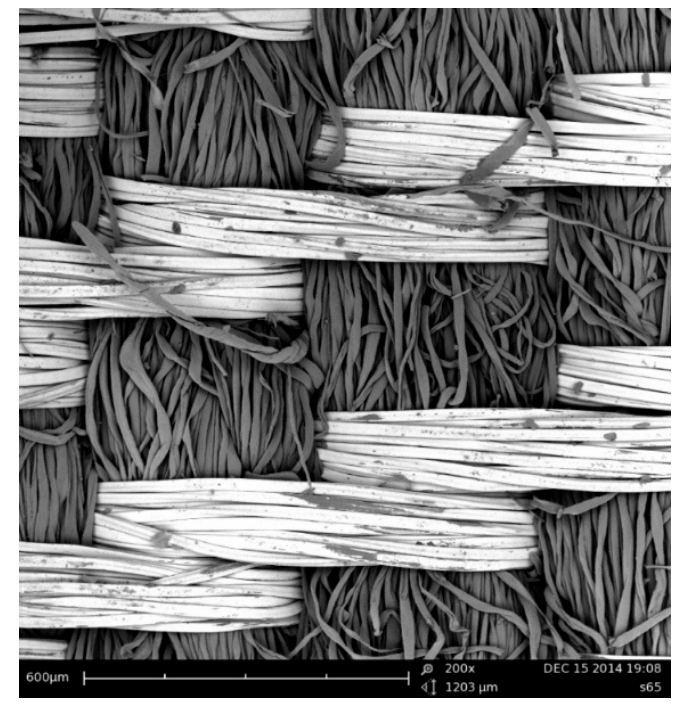

b) Irregular Twill structure

Fig. 1. SEM image of fabrics under test 

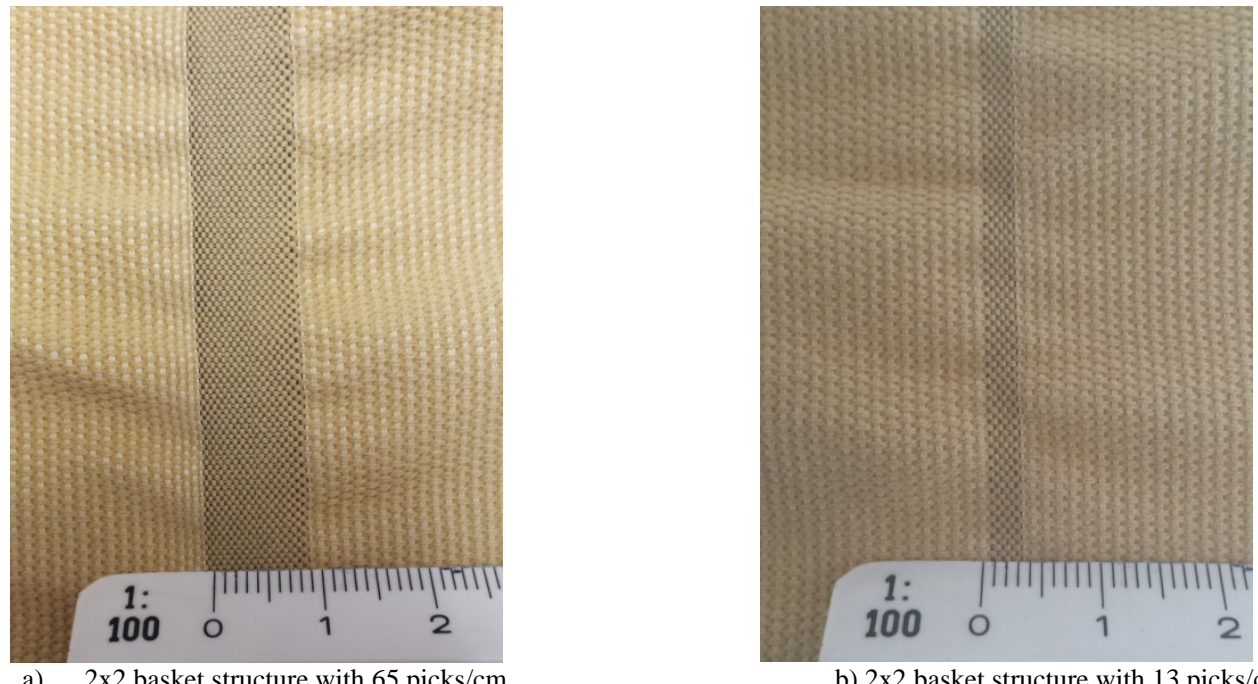

b) 2x2 basket structure with 13 picks/cm

Fig. 2. Photograph of elaborated fabrics 


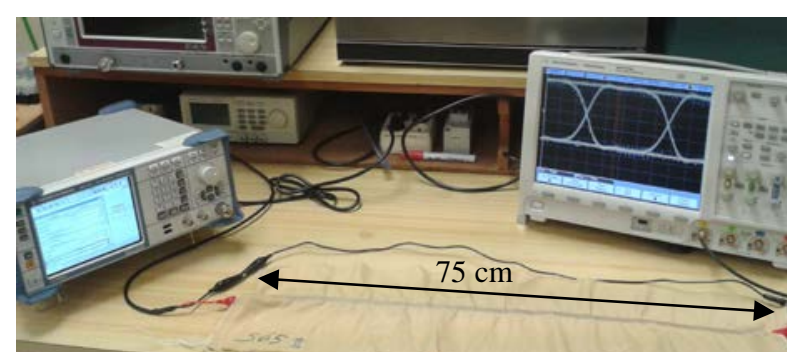

Fig. 3. Photograph of experimental setup to evaluate the eye diagram.

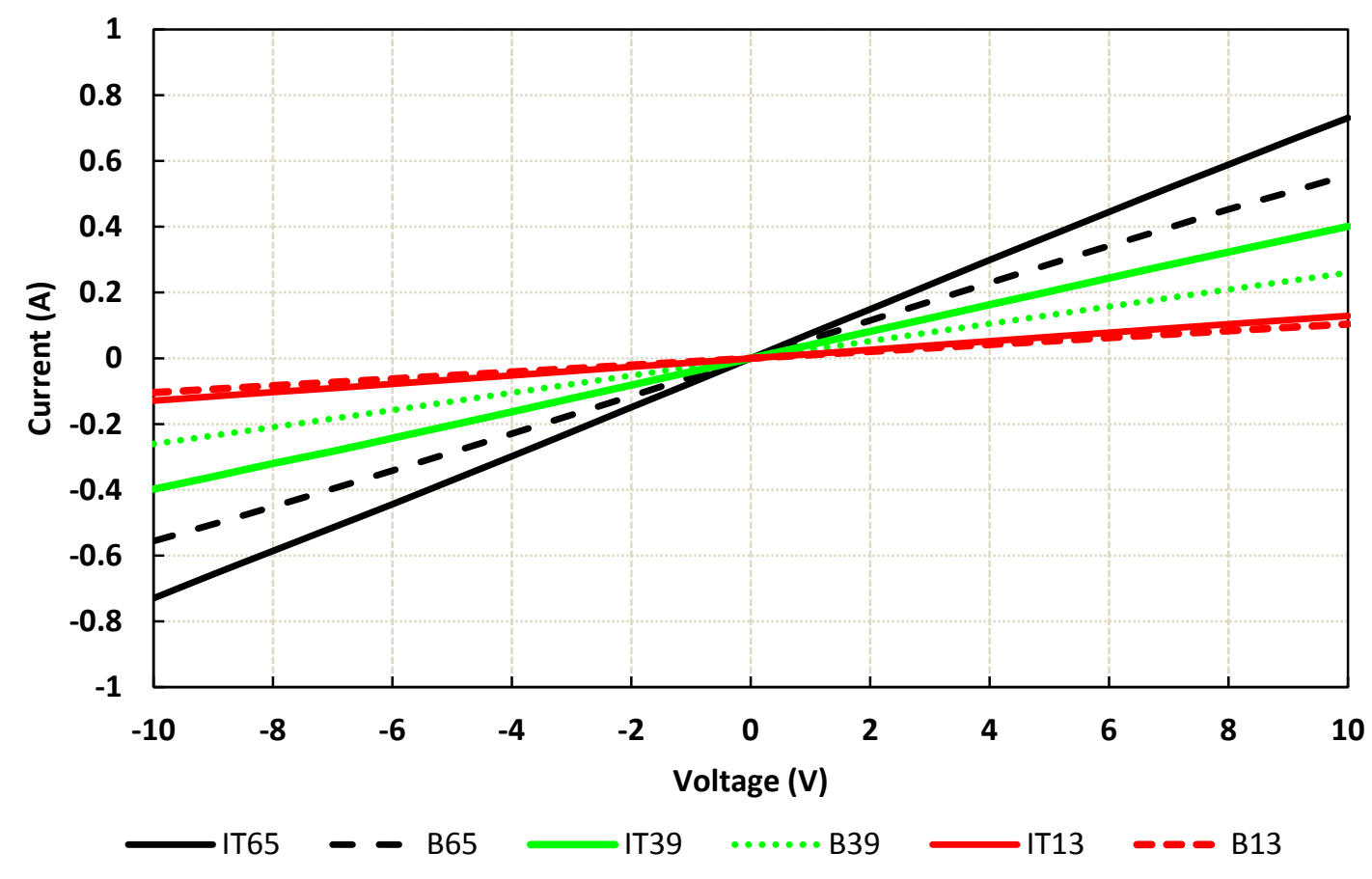

Fig. 4. DC Voltage current characteristic of FUT 


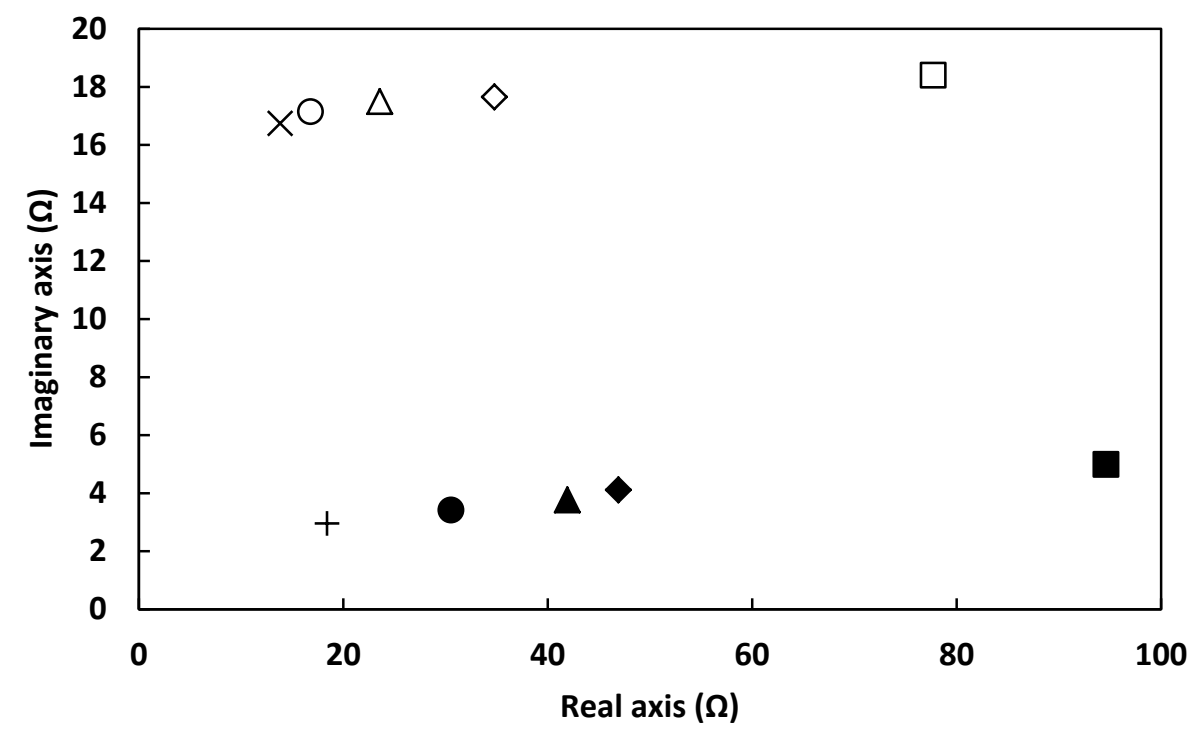

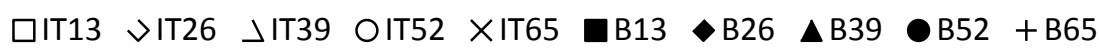

Fig. 5. Real and imaginary impedance of FUT at $1 \mathrm{MHz}$ 


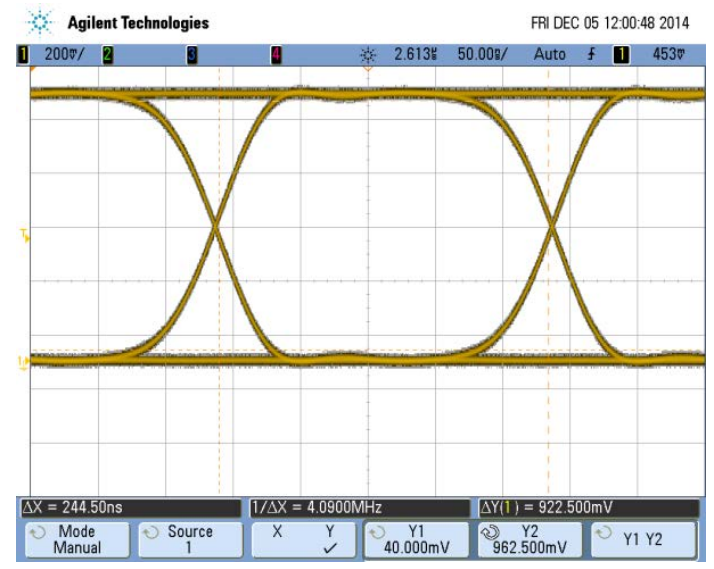

a)
A Agilent Technologies

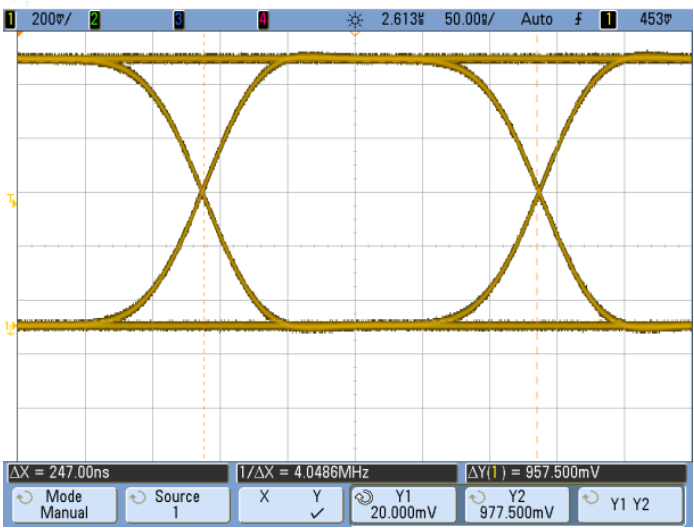

FRI DEC 05 11:08:21 2014 Agilent Technologies

b)

THU DEC 11 18:29:02 2014

Agilent Technologies 1] $2000 /$ 口

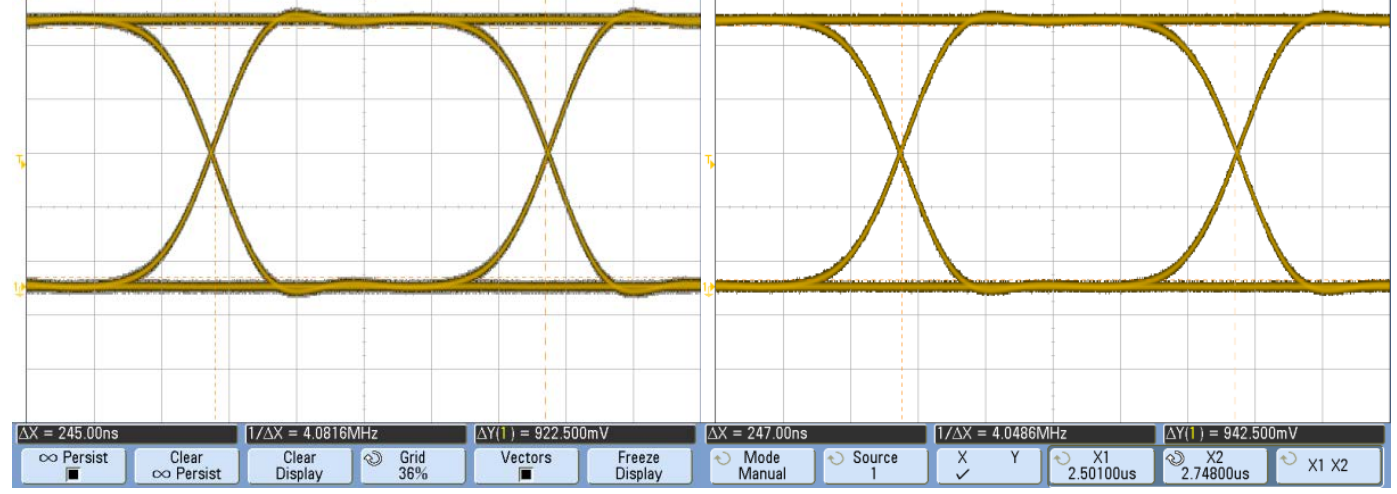

c)

d)

Fig. 6. Eye diagram a) IT13 b) B13 c) IT65, d) B65 
Table 1. Fabrics under test

\begin{tabular}{|l|l|l|l|}
\hline FUT & Structure & Picks & Theoretical \\
{$[\mathrm{p} / \mathrm{cm}]$} & Thickness [mm] \\
\hline B65 & $2 / 2$ Basket & 65 & 10 \\
\hline B52 & $2 / 2$ Basket & 52 & 8 \\
\hline B39 & $2 / 2$ Basket & 39 & 6 \\
\hline B26 & $2 / 2$ Basket & 26 & 4 \\
\hline B13 & $2 / 2$ Basket & 13 & 2 \\
\hline IT65 & Irregular Twill & 65 & 10 \\
\hline IT52 & Irregular Twill & 52 & 8 \\
\hline IT39 & Irregular Twill & 39 & 6 \\
\hline IT26 & Irregular Twill & 26 & 4 \\
\hline IT13 & Irregular Twill & 13 & 2 \\
\hline
\end{tabular}


Table 2. FUT resistance and inductance per unit length

\begin{tabular}{|c|c|c|c|c|c|c|c|c|c|c|}
\hline & IT13 & IT26 & IT39 & IT52 & IT65 & B13 & B26 & B39 & B52 & B65 \\
\hline $\mathbf{R}(\mathbf{\Omega} / \mathbf{c m})$ & 1.04 & 0.46 & 0.31 & 0.22 & 0.18 & 1.26 & 0.63 & 0.56 & 0.41 & 0.25 \\
\hline $\mathbf{L ~ ( n H / c m )}$ & 39.07 & 37.47 & 37.13 & 36.40 & 35.53 & 10.60 & 8.73 & 8.00 & 7.27 & 6.27 \\
\hline
\end{tabular}


Table 3. Eye diagram parameter at $4 \mathrm{MHz}$

\begin{tabular}{|c|l|l|}
\hline Performance & EH (mV) & EW (ns) \\
\hline IT13 & 922.5 & 244.5 \\
\hline IT65 & 922.5 & 245.0 \\
\hline B13 & 957.5 & 247.0 \\
\hline B65 & 942.5 & 247.0 \\
\hline
\end{tabular}

